\title{
"Stylization" and "Spirit Presentation through Appearance"-on Portrait Techniques of Ming and Qing Dynasties
}

\author{
Tianyu Liu \\ Chengdu Academy of Fine Arts, Chengdu, 610500, China
}

\begin{abstract}
Keywords: stylization, Present spirit through appearance, Portrait
\end{abstract}
\begin{abstract}
Chinese portrait has accumulated rich creation experiences in the long-term evolution process and forms abundant and complete presentation models. The ancients summed up their common laws to maximize the performance of the presentation language. The portraits ushered in the most brilliant generation in the Ming and Qing Dynasties. The stylization functions of portraiture technology are analyzed in the paper to complete the analysis of "appearance" and "spirit", "common features" and "personality" to realize the roles change "stage" of the reality and the ideal image. It also explores more feasibilities for the development of traditional painting Chinese.
\end{abstract}

The word "portrait" of China comes from the western painting terms. Although there are the portraits in our traditional painting, there is no exact term of the "portrait" in the relevant theory. The word "portrait" in Cihai is interpreted as "the specific characters of painting, including head image, length portrait group images, which is required to achieve the unity of form and spirit realm." In this kind of traditional painting, this category is called "portraiture", "vivid" or "portrait"". Chinese portrait has accumulated rich creation experiences in the long-term evolution process and forms abundant and complete presentation models. The ancients summed up their common laws to maximize the performance of the presentation language. The portraits ushered in the most brilliant generation in the Ming and Qing Dynasties. The stylization functions of portraiture technology are analyzed in the paper to complete the analysis of "appearance" and "spirit", "common features" and "personality" to realize the roles change "stage" of the reality and the ideal image. It also explores more feasibilities for the development of traditional painting Chinese.

The development of portrait painting in Ming Dynasty has not been satisfied with the original performance skills, followed by the traditional "stylized" techniques gradually enriched and improved. It laid the foundation for the prosperity of the Ming and Qing Dynasties. Zhang Geng in Qing Dynasty wrote a book named "country painting record" to say "the sign is a portrait of two factions, heavy ink, ink is then deposited into the bone, color, and color of the spirit has long ink in the bone will the sensitive Bo Chen (Zeng Jing) learned. A little with the ink, tick the facial parts with full effect, color rendering, the Jiangnan transfer method". From the two painting can be seen, whether it is heavy or heavy ink, color is in the development of the yuan on the basis of drawing portrait, painted facial features in terms of performance, are keeping a traditional "program" techniques, but still does not lose its sense of reality, is described as "a wonderful look, like a mirror and shadows." It can be seen that the "stylization" and "spirit presentation through appearance " do not contradict.

The representative of the "ink" school is the portrait of the Bo Chen painting school represented by Zeng Jing.

Zeng Jing and his Bochen school wrote a Chinese portrait painting whcih is undoubtedly the densest. But in some of the research literature of art history, such as the "China art history" by Dachunxiya and "Chinese painting history" by Zheng Wuchang that Zeng Jing portrait achievements "is a reference for the absorption process, today, there are still many such arguments. After reading the relevant historical data and analysis of works, the portraits not only did not participate with ziphah, and has a strong tradition of "stylized" techniques of color. 
The author went to the Zhejiang Museum for many times to do the research work of the great works of Zhang Qingzi's Image. It was made in 1622, with the maturity of representative works, the original is printed, the techniques clearly appear, face directly to harmonious color, bright feeling, with a slight change in the local natural color, has a certain concavity, factors but not "changing light dark Yangming". These are in line with the general program of traditional portraits, facial contour lines accurately, and then melt hidden color, along with thin layer rendering. The inherent concavity, then the color, the layers of natural light, between the pen and gel pen and ink marks, with local color, the soft colors. The Song Zongqian in Qing Dynasty said ink and color ink marks according to the essay, state, after the view, non-color ink on the surface of it is not, the color of God" ("Mustard boat made learn painting perfectly). In addition, the Nanjing Museum. It is light ink outline features, along with natural light to light, light tan ink dye, repeated several times, the ink does not cover the bone. I was extremely uneven light, natural clear, moist complexion. Thin clothes smooth outline, great tension, with sparse Dang, is "not keep" charm, with ancient law, show the right finger and toe under visible, showing in an easy-going Gu temperament, and this kind of expression is a characteristic Zengjing portrait painting, literati aesthetic taste that Zengjing portrait. Zhang Qingzi Image, Tsang's other works. Therefore, in the above analysis conclusion can be had after the whale works, it should be with a view to lighting change "Western painting" is not "absorption and reference", but with some strong "stylized" folk painters to color my dyed color and concave convex method. He is good at absorbing and development of folk art "stylized". His works can be an inspiration of the development of Chinese traditional painting art.

The "color" faction, is generally mature in the Ming and Qing Dynasties in the late Yuan in people pay attention to "ink bone" and "light blaster", this painting is based on the use of ink, coloring and shading etc., so as to further strengthen. The Handed down works is hidden in the Nanjing Museum of Ming Dynasty, the Ming Dynasty portrait album, the most prominent achievements, the most obvious color characteristics. Start "Ming portrait album", the twelve characters were sound true to life characters, skin, moustache is elastic texture. The picture in a unified style, unified and from each character's forehead reflects the different temperament and personality. Although the authors focus on the use of color ink and pen, but for bone have attached great importance to the Qing Jiang Ji ", baking, masonry," four pen to perfect. The features of the people and the structure, skeleton, are painted with light according to the inherent structure, although only a few pens, but quite accurate, some facial structure based on light ink line to "bake", such techniques can be found to accord with the writings discussed in Shen Zongqian et al. With the color is the expression of the character of the skin of the reality, the technique of rain Yuan Wang Shu "writing like secret" in the "painting" is basically the same, but according to each person's skin color changes slightly adjusted to make the characters more vivid. The character of the wind, the degree of rhyme mainly to grasp the shape of the performance, that is, the shape is coming".

"Ming portrait album" presented the characters' faces and features the characters of "rich, lean, white and extravagant" and the "composition program very much like" (similar to the modern "passport"). Each person's face, first of all is to see his wet, thin, then pay attention to facial features. In the portrait book, such as Li Rihua's "rich", Liu Xianchong's "lean", are portrayed very wonderful, personality characteristics are very prominent. However, the twelve "Feng" and "lean" face is different, but the "phase" technique is much the same: the artist featured amount, nose, chin three parts, depicting the ears and cheekbones with ancient maps, and fixed form "information is large, the ups and downs of the bones out wrinkles or looming, then the shape and the spirit were both coming. The face is always "eight" will according to the features of other features also has a strong "stylized" factors. However, these did not prevent the artist to express their different charm style, the screen is not suspected of stagnation, rigid sense. It is because of good grasp of "program "and specific images. The relationship between the success of the performance of the spirit of character, temperament and character, only to produce "Ming" this book portrait "is the color of the Chinese traditional portrait masterpiece.

Of course, portrait works far more than a few, and at the same time Zengjing portrait painter in Qing Dynasty edited by Xu Qin "Ming painting" in the record of not more than ten people, which is 
representative of Cui Zizong and Chen Hongshou, according to the "Ming painting" cloud: "chase ancient paintings Cuishi figure out. But it is worth mentioning that there are kinds of clever structure, transform the thing in personality in the wild and intractable is known, but the style is deliberately chase ancient". Single handed down from ancient portraits, the composition for the traditional "stylized" "pictures" or supplement the landscape of the aesthetic taste of literati friends Figurine, ancient method, has become a distinct characteristic of Chen. He used the photo method described in line for facial bone; traditional clothing lines outline blend together, who is similar to Zengjing "Jiangnan school transfer", as they handed down a self-portrait of the angle, and Ming Dynasty "painting" three nine like exactly the same, and this kind of works in the angle of Chen's portrait works handed down more clearly. The distinctive style of Chen Laolian is the development from the traditional "stylized" system.

The portrait of Qing Dynasty is mature in techniques and styles. The representative painters included Xu Zhang, Gao Ding, Yu Zhiding and Ren Yi. However, there is a painter who cannot be avoided. He has a special significance in the history of Chinese portrait painter of the Qing Dynasty named Lang Shining.

If Zengjing did not cover the bone with Western irrelevant words, Lang Shining has become the representative of Chinese and Western painting fusion characters due to the special identity of Chinese Westerners body by traditional painting tools, In one of his masterpiece "Qianlong Royal map" for example, works with anatomic structure as a means of expression, focusing on stereoscopic vision, no ink stick body, the bone, the role of performance in the first place, also failed to shake off the shadow of the object. Although in order to comply with the Chinese traditional aesthetic and weakens the contrast of light and shade, but in the lines of the clothes and accessories in order to show the texture while retaining a reflective, so that scholars can participate in the Qing Dynasty. Zou Yigui also waked up the method without writing. Although the industry is not the carpenter, into the painting ", has given Lang Shining Qianlong realistic ability to let him paint the face let China painter clothes and background of the historical records, the British people to see the paintings of Lang Shining Barlow, that" the picture is exquisite, but too trivial. Lang Shining painting and gone similar to European painting......" It can be seen that not only Lang Shining's traditional Chinese painting is not accepted by the Chinese traditional aesthetics. Even the European painters did not agree with it.

As the light with physiological structure of Lang Shining's painting was not consistent with the traditional painting and other Chinese intention to traditional aesthetic style line shape, the use of China traditional painting form the Xu Zhang portrait painter and Chinese folk will undoubtedly have a greater influence.

Xu Zhang was located in Songjiang Lou County (now Lou County in Jiangsu). His painting style also conformed to Bochen School". "The map of Songjiangbangyan" was handed down. It began in Songjiang famous scholar of the male thinking, stop at the door of Chen Zilong, a total of one hundred and ten people, the existing ninety-four people, is now in the possession of the Nanjing museum. Although the former is depicted as mostly on portraits and legends and historical materials and pictures, but after Xu's personal emotional insights into, not only for - Shaw, tried to figure in the expression, satisfaction, temperament and other aspects of different temperament to accomplish something, it should be said that there are many works have reached the spirit the realm of meaning. According to the relevant historical records: Xu's portrait, with light ink for bone, draw facial features, and then to Tan Son ink smudge facial color with pale face bump, with the back cover, thin powder, to strengthen the hidden pen marks bump, and finally to light ochre, rouge complex hook features, with ink on the eyebrow, wake up the eyes, hair color has reached reconcile, and Yuan Wang Duo "written records like secret": the powder cover is thin, dry ink Tan is the same, the difference is developed on the basis of Xu Zhang, finally to light Yunran repeatedly, in order to achieve the "state of the ink in ink color mark essays." Xu Zhang and pen in the clothing on the same face, writing does not go beyond the "eighteen." category, but also not wedded to a, on hand foot portrayals than previous moreprecise, but also to the environment has to reflect the inner world of characters, although none of the above to get rid of the traditional painting China "program" the way, according to the "Lou county" also recorded on Xu's first with rice paper as portrait, no doubt in all aspects have greatly 
increased the difficulty of control, although the Ming and Qing Dynasties two generations each artist each has a different style, but there are also common creation rules to be followed, "program" remarkable influence, therefore and from the results that, during the two dynasties portrait painter works are completed based on "stylized". Wu Daozi's ink for bone covered with light color. Li Jiaxiang was still the mainstream aesthetic in that time.

It can be said that due to the "stylized" factors, the painting technology in China traditional painting became relatively simple. It was easy to grasp its regularity, which greatly increased its maneuverability, as the painter provides a complete method to present spirit through appearance, the combination of generality and personality to realize the transformation stage of real person and ideal image.

\section{References}

[1] Zhu Wanzhang, On the Aesthetic Value of Portraits in Ming and Qing Dynasties [J]. Journal of Jilin University of Arts, 2009(3): 17-20.

[2] Li Xiuxia, Female Portraits of the Ming and Qing Characteristics and Cultural connotations [J]. Arts Exploration, 2010, 24(5): 14-15+22.

[3] Li Xiaofang, Portraits in Ming Dynasty: The Immortal Arts [J]. Journal of Central South University of Forestry \& Technology (Social Sciences), 2011, 5(6): 153-154.

[4] Tang Shaofeng, Disscusion on the Impact of Western painting upon Chinese portraits during the Ming Dynasty and Qing Dynasty [J]. Arts Exploration, 2007, 21(4): 19-20. 